\title{
Diagnostic accuracy of squash cytology and role of GFAP immunoexpression in glial tumors
}

\author{
Sanjeev Kishore', Brijesh Thakur ${ }^{2, *}$, Aparna Bhardwaj', Anuradha Kusum ${ }^{4}$ \\ ${ }^{1}$ Professor \& HOD, ${ }^{2}$ Associate Professor, ${ }^{3}$ Professor, Dept. of Pathology, Shri Guru Ram Rai Institute of Medical \\ $\&$ Health Sciences, Dehradun, Uttrakhand, ${ }^{4}$ Professor, Dept. of Pathology, Swami Rama Himalayan University, \\ Dehradun, Uttrakhand, India \\ *Corresponding Author: \\ Email: drbrijeshthakur03@gmail.com
}

\begin{abstract}
Introduction: Intraoperative squash technique provides rapid and reliable diagnosis which is helpful to monitor or modify the treatment approach. We carried out the present study to compare the accuracy of squash preparation with histopathological diagnosis and also to study the role of GFAP immunostaining in gliomas.

Material and Methods: Clinicoradiologically diagnosed 127 cases of gliomas were subjected to both squash smear cytology and histopathological examination; also to GFAP staining using monoclonal antibody (EP672Y clone). Taking histopathology as gold standard, diagnostic accuracy and statistical values were calculated. Immunohistochemical analysis was compared with final diagnosis.

Results: Overall diagnostic accuracy of squash cytology was observed as $95.2 \%$ (sensitivity=99.1\%, PPV= 99.1\%). All low grade astrocytomas and ependymoma showed strong diffuse positivity while high grade astrocytomas were focally reactive. Negative immunoexpression was seen in case of oligodendroglioma and pituitary adenoma.

Conclusion: Squash cytology is of great value in rapid intraoperative consultation of gliomas. GFAP can be used as an adjunctive tool to verify the astroglial differentiation especially in diagnostically difficult cases.
\end{abstract}

Keywords: diagnostic accuracy, GFAP, Gliomas, Squash.

Received: $15^{\text {th }}$ May, 2017

Accepted: $14^{\text {th }}$ October, 2017

\section{Introduction}

Gliomas are the frequent primary brain tumors accounting for about $81 \%$ of malignant brain tumors and include astrocytomas, oligodendrogliomas, oligoastrocytomas, ependymomas and mixed neuronal glial tumors. ${ }^{1,2}$ An accurate diagnosis of these lesions is necessary for therapeutic and prognostic reasons. As radiological features may not always be conclusive about etiology of the lesions, rapid intraoperative cytological diagnosis of lesions by squash smear technique is an useful adjunct technique to help the operating surgeon to come to a correct decision regarding the further management of the patient. ${ }^{3}$

Some diagnostic problems such as recognition of highly differentiated astroglial neoplasm and differential diagnosis between poorly differentiating tumors and metastasis to CNS have been reported. ${ }^{4,5}$ In such cases immunohistochemistry may be regarded as essential tool for more precise identification of diagnostically difficult gliomas. Glial fibrillary acidic protein (GFAP) can be used as a specific marker for immature, reactive, neoplastic astrocytes and ependymal cells. ${ }^{6}$ The aim of our study was to compare the accuracy of squash preparation with histopathological diagnosis and to study the role of GFAP as well as the pattern of GFAP immunostaining in gliomas.

\section{Material and Methods}

The present study was conducted in department of Pathology for a period of three years in which we included total one hundred twenty seven cases of clinically and/or radiologically diagnosed gliomas. Approval of the study was taken from the institutional ethical committee. Intraoperative biopsy samples were sent immediately to cytology section in isotonic saline for processing along with relevant clinicoradiological and peroperative findings. Gross and naked eye evaluation was done for all specimens.

Smears were prepared after removing extra saline and using the 1-2 mm. of tissue piece of representative area with the help of edge of sterile blade. After placing the tissue piece at one edge of clean dry slide, it was crushed with another slide with slight uniform pressure to spread it into thin film ideally to achieve a monolayer of cells. Two smears were immediately fixed in 95\% ethanol and stained with H\&E and Papanicolaou stain. Additional 
slides were air dried and stained with MayGrunwald Giemsa stain. All smears were examined to evaluate cellularity and cytomorphological features. Tissue biopsy at the end of surgery was fixed to $10 \%$ buffered formalin and subjected to paraffin embedded sectioning for histopathology. Cytological diagnosis was correlated with histopathological diagnosis. Taking histopathology as gold standard, diagnostic accuracy and statistical values were calculated using Diagnostic \& Agreement statistics software.

Two micron thick sections were taken for GFAP staining using monoclonal antibody (EP672Y clone by cell marque: Key codeCMC25831020) and cytoplasmic immunopositivity was seen as dark brown colour on bluish background (counterstaining with Harris's hematoxylin). Positive results were divided according to staining intensity as strongly/weakly positive and pattern as diffuse/focal. Immunohistochemical analysis was compared with final diagnosis.

\section{Results}

In our study, most of the patients presented with headache, nausea, vomiting, seizure and tinnitus. The age of the patients varied from 11 to 70 years. Maximum number of patients
(29.9\%) was observed in 41-50 years of age group. Male:Female ratio was 1.95:1. Cerebral hemisphere located gliomas were observed in about $48.5 \%$ of all cases. Frontal lobe was the most common site followed by overlapping cerebral lesions, temporal, parietal, posterior fossa, suprasellar and ventricular regions. On squash cytology, 04 cases were considered as benign lesions comprising 02 cases of mixed neuronal glial tumors and 02 inconclusive cases. Rest of the 123 cases diagnosed as astrocytomas, GBM, oligodendroglioma, ependymoma and were taken as malignant lesions. Cytological examination revealed $76.4 \%$ cases of astrocytomas followed by ependymoma (8.6\%), oligodendroglioma(6.3\%), GBM(5.5\%), mixed neuronal glial lesions(1.6\%) and inconclusive cases $(1.6 \%)$.

On subsequent histopathological examination, out of 97 cytologically diagnosed astrocytomas, 86 cases were diagnosed as astrocytomas (excluding grading deviations), 06 cases as oligodendroglioma, 04 cases as ependymoma and 01 case as GBM. Diagnostic accuracy of squash cytology was observed $88.6 \%$ in cases of astrocytomas. Out of 07 cases of GBM on squash cytology, 06 cases of GBM and 01 case of gliosarcoma were diagnosed on histopathology, giving diagnostic accuracy of $85.7 \%$ (Table 1).

Table 1: Comparative analysis of squash cytology and histopathology in gliomas

\begin{tabular}{|c|c|c|c|c|}
\hline $\begin{array}{l}\text { Cytological } \\
\text { diagnosis }\end{array}$ & $\begin{array}{l}\text { No. of } \\
\text { cases }\end{array}$ & Histopathology & $\begin{array}{l}\text { No. of } \\
\text { cases }\end{array}$ & $\begin{array}{c}\text { Diagnostic } \\
\text { Accuracy }(\%)\end{array}$ \\
\hline \multirow{4}{*}{ Astrocytoma } & \multirow{4}{*}{$97(76.4 \%)$} & Astrocytoma grade I, II \& III & 86 & \multirow{4}{*}{$88.6 \%$} \\
\hline & & $\begin{array}{l}\text { Oligodendroglioma grade I, } \\
\text { II \& III }\end{array}$ & 06 & \\
\hline & & Ependymoma & 04 & \\
\hline & & GBM & 01 & \\
\hline \multirow[t]{2}{*}{ GBM } & \multirow{2}{*}{$07(5.5 \%)$} & GBM & 06 & \multirow{2}{*}{$85.7 \%$} \\
\hline & & Gliosarcoma & 01 & \\
\hline \multirow{2}{*}{$\begin{array}{l}\text { Oligodendroglioma } \\
\text { I, II \& III }\end{array}$} & \multirow[t]{2}{*}{$08(6.3 \%)$} & $\begin{array}{l}\text { Oligodendroglioma grade I, } \\
\text { II \& III }\end{array}$ & 07 & \multirow[t]{2}{*}{$87.5 \%$} \\
\hline & & Pituitary adenoma & 01 & \\
\hline \multirow[t]{2}{*}{ Ependymoma } & \multirow{2}{*}{$11(8.6 \%)$} & Ependymoma & 09 & \multirow{2}{*}{$81.8 \%$} \\
\hline & & Astrocytoma & 02 & \\
\hline \multirow[t]{2}{*}{$\begin{array}{l}\text { Mixed neuronal } \\
\text { glial tumors }\end{array}$} & \multirow[t]{2}{*}{$02(1.6 \%)$} & $\begin{array}{l}\text { Dysplastic cerebellar } \\
\text { Gangliocytoma }\end{array}$ & 01 & \\
\hline & & Ganglioglioma & 01 & \\
\hline \multirow{2}{*}{$\begin{array}{l}\text { Inconclusive/no } \\
\text { definite opinion }\end{array}$} & \multirow{2}{*}{$02(1.6 \%)$} & Reactive gliosis & 01 & \\
\hline & & Astrocytoma & 01 & \\
\hline
\end{tabular}

From 08 cases of oligodendrogliomas on cytology, final diagnosis was correlated in 07 cases and 01 case of pituitary adenoma was diagnosed. Diagnostic accuracy was calculated $87.5 \%$ irrespective of the grading of oligodendrogliomas. In 11 cases of cytologically diagnosed ependymoma cases, 09 cases of ependymoma and 02 cases of astrocytoma were considered as final diagnosis on histopathology. Diagnostic accuracy was $81.8 \%$ (Table 1). Taking histopathology as reference method, 
overall diagnostic accuracy was $95.2 \%$. Both sensitivity and positive predictive value was calculated as $99.1 \%$ (Table 2).

Table 2: Statistical values of squash cytology taking histopathology as gold standard

\begin{tabular}{|c|c|c|c|c|c|c|c|c|}
\hline & Squash & \multicolumn{2}{|c|}{$\begin{array}{c}\text { Final } \\
\text { Diagnosis }\end{array}$} & Sensitivity & Specificity & PPV & NPV & $\begin{array}{c}\text { Diagnostic } \\
\text { accuracy }\end{array}$ \\
\hline & & B & $\mathbf{M}$ & \multirow{3}{*}{$99.1 \%$} & \multirow{3}{*}{$75 \%$} & \multirow{3}{*}{$99.1 \%$} & \multirow{3}{*}{$75 \%$} & \multirow{3}{*}{$95.2 \%$} \\
\hline $\begin{array}{l}\text { Malignant } \\
\text { (M) }\end{array}$ & 123 & 01 & 122 & & & & & \\
\hline $\begin{array}{l}\text { Benign } \\
\text { (B) }\end{array}$ & 04 & 03 & 01 & & & & & \\
\hline
\end{tabular}

All cases were subjected to GFAP immunostaining and were analyzed for staining results in different areas of the lesions. All cases of astrocytoma grade I, II and ependymoma showed strong diffuse positivity. Out of 37 cases of astrocytoma grade III, 33 cases revealed weak diffuse positivity and 04 cases showed focal strong positivity. Diffuse weak positivity with few unstained areas was seen in 06 cases of grade IV astrocytoma while strong positivity of few cells noted in 02 cases. Piloid areas of pilocytic astrocytoma showed strong positivity in 05 cases. Weak positivity was observed in one case each of mixed neuronal glial tumor and reactive gliosis. All oligodendrogliomas and pituitary adenoma showed negative GFAP staining (Table 3).

Table 3: Comparative analysis of GFAP immunoexpression with final diagnosis

\begin{tabular}{|c|c|c|c|}
\hline Final Diagnosis & $\begin{array}{l}\text { No. of } \\
\text { cases }\end{array}$ & GFAP immunoexpression & $\begin{array}{l}\text { No. of } \\
\text { cases }\end{array}$ \\
\hline Astrocytoma grade I & 17 & Diffuse strongly positive & 17 \\
\hline \multirow{2}{*}{ Pilocytic astrocytoma } & \multirow[t]{2}{*}{07} & Strongly positive (piloid area) & 05 \\
\hline & & Weak positive & 02 \\
\hline Astrocytoma grade II & 28 & $\begin{array}{l}\text { Strongly positive in diffuse } \\
\text { pattern }\end{array}$ & 28 \\
\hline \multirow[t]{2}{*}{ Astrocytoma grade III } & \multirow[t]{2}{*}{37} & Strongly positive in focal areas & 04 \\
\hline & & Weak positive in diffuse pattern & 33 \\
\hline \multirow[t]{2}{*}{ Astrocytoma grade IV } & \multirow[t]{2}{*}{08} & Strongly positive in some cells & 02 \\
\hline & & $\begin{array}{l}\text { Diffuse weak positivity with } \\
\text { few unstained areas }\end{array}$ & 06 \\
\hline Ependymoma & 13 & Strongly positive & 13 \\
\hline Oligodendroglioma & 13 & Negative & 13 \\
\hline Pituitary adenoma & 01 & Negative & 01 \\
\hline Mixed neuronal glial tumors & 02 & Weak positive & 01 \\
\hline Reactive gliosis & 01 & Weak focal Positive & 01 \\
\hline
\end{tabular}

\section{Discussion}

Eisenhardt and Cushing introduced intraoperative cytological investigations in the 1930s for rapid examination of neurosurgical specimens. Intraoperative consultation is usually requested to differentiate neoplastic from reactive lesions; to separate metastatic lesions from primary tumors; to determine the grading and margins of the tumor. ${ }^{7}$ The smear technique can provide a rapid, reasonably accurate and reliable diagnosis to the operating surgeon. In case of very tiny tissue bit, it is possible to evaluate cellular details and to give definite pathologic diagnosis by the smear technique. Frozen section may lead to ice crystal artifacts in brain tissue which is more challenging for pathologist. The disadvantage of smear technique lies with those lesions that are too firm to smear like meningiomas, schwannomas or metastatic deposits but these are responsible for a small number of lesions. Also squash technique has limited advantage in evaluation for margin status. $^{8}$

In the present study, overall diagnostic accuracy of squash cytology was $95.2 \%$, which is comparable to other studies as well. ${ }^{8-12}$ Rani et al. and Jha et al. found accuracy of squash cytology as $93.63 \%$ and $82.35 \%$ respectively in intraoperative diagnosis of CNS lesions. ${ }^{8}$ Imtiaz et al. observed an overall diagnostic accuracy of crush smear of $93.5 \%$ in a cross sectional study including 100 intraoperative neurosurgical biopsies. ${ }^{11}$ 
Although biopsy submitted in all cases was adequate grossly, however in some cases discrepency was noted in cytological and histological diagnosis. There were 10 cases considered as astrocytomas on squash cytology which turned out to be oligodendroglioma (06 cases) and ependymoma (04 cases) on histopathology. This was due to presence of large nuclei and clustering around blood vessels as well as lack of uniform nuclei in smears of oligodendroglioma which gave false impression of high grade astrocytoma. Likewise, vascular proliferation, increased number of fibrillary astrocytes and absence of rosettes might be the reason of erroneous interpretation of astrocytoma. Lack of representative biopsy for squash may be the reason for misinterpretation of the cases of GBM and gliosarcoma because of the absence of necrosis, vascular proliferation and sarcomatous component in the smears examined. So, grading of malignancy should be commented on a permanent section after an optimal evaluation of the entire sample in the absence of unequivocal features of glioblastoma. The misdiagnosis of ganglioglioma was due to the poor preservation and absence of ganglion cells in smears. One case of pituitary adenoma was misinterpreted as oligodendroglioma because of monotonous cell population, consideration of focal edema as perinuclear halo and ectopic site of tumor. Also astrocytoma is major differential diagnosis for ependymoma and they may be misinterpreted interchanageably. But these two need to be distinguished as they may belong to different grades. Two cases were found to be inconclusive due to sampling error, crush artifact, very low cellularity and more fibrosis. Some of the other authors also described various errors during introperative consultation which can help in providing a more conclusive opinion to the operating surgeon. , $^{2,13-16}$

Distinguishing cytomorphological features of gliomas are not always straightforward, so reliable immunohistochemical specific proteins of glial differentiation may contribute greatly to increase the diagnostic accuracy for difficult glial tumors. The most important and invaluable consistently expressed marker of glial differentiation is intermediate filament glial fibrillary acidic protein. GFAP was originally isolated from old multiple sclerosis plaques and subsequently localised by immunoflorescence in fibrillary astrocytes. GFAP currently used as routine antigenic marker for normal, developing and maturing astroglial cells as well as pathologically altered astrocytes and ependymal cells. ${ }^{17}$

In our experience from this study, fibrillary or piloid areas of pilocytic astrocytoma and grade I astrocytoma showed diffuse strong GFAP positivity while rosenthal fibres were found to be negative (Fig 1a \& 1b). Similar features were decribed by Valesco et al in low grade astrocytomas. ${ }^{18}$ Rosenthal fibres stained either as solid masses only showing peripheral thin rim or totally devoid of immunoreactive material were observed. Also noted eosinophilic hyaline bodies outlined as dense immunoreactive material in astrocytomas.

In cases of grade II diffuse astrocytoma, cell processes of fibrillary astrocytomas cells were strongly immunoreactive for GFAP while the perinuclear cytoplasm was less strongly stained. Cells with gemistocytic appearance were found to be strongly GFAP positive (Fig 1c\&d). GFAP immunostaining revealed moderate to strongly positive uni or bipolar cells in grade III astrocytomas while large bizzare multinucleated cells were negatively stained Cells with gemistocytic appearance were found to be strongly GFAP positive (Fig 1e\&f). Chen et al. also revealed same opinion about the GFAP staining pattern in grade II \& III astrocytomas. ${ }^{(19)}$ In highly anaplastic astrocytoma, only a few scattered cells were immunoreactive Cells with gemistocytic appearance were found to be strongly GFAP positive (Fig $1 \mathrm{~g} \& \mathrm{~h}$ ). In this study, elongated and bipolar cells in glioblastoma (grade IV astrocytomas) showed strong positive reaction while small round cell component revealed little or no staining. The areas of necrosis and macrophages remained unstained. Similar features have been described by Eng LF and Rubinstein LJ. ${ }^{(20)}$ 


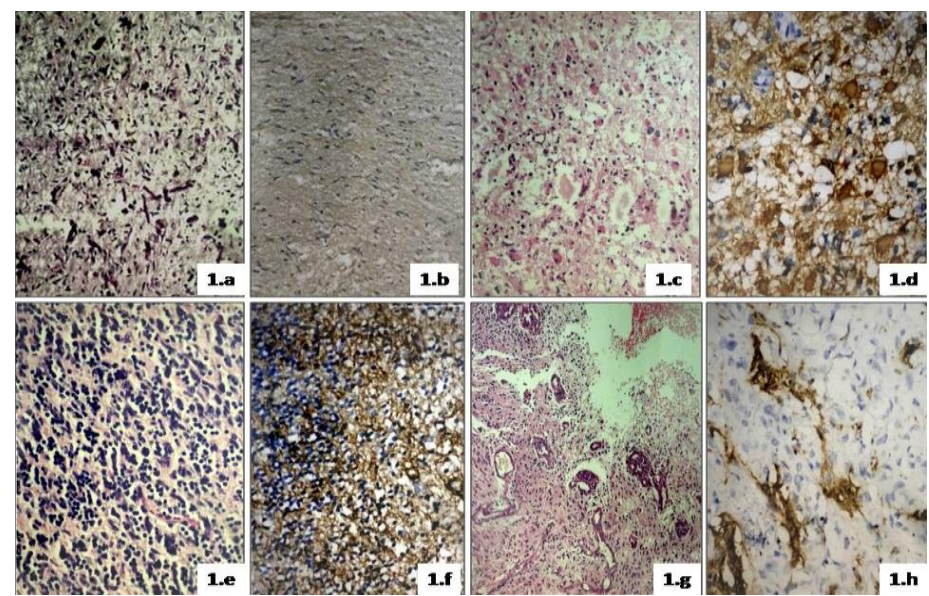

Fig. 1: a: Pilocytic astrocytoma showing rosenthal fibres and eosinophilic bodies $(\mathrm{H} \& \mathrm{E}, 200 \mathrm{X})$; b: Pilocytic astrocytoma showing GFAP positivity in cytoplasmic processes of pilocytes (GFAP, 100X); c: Low grade astrocytoma showing edema, microcystic changes and many gemistocytes $(H \& E, 200 X)$; d: Low grade astrocytoma showing moderate GFAP positivity in gemistocytes and their processes (GFAP, 400X); e: Grade III astrocytoma showing high cellularity, nuclear pleomorphism and proliferation of thin walled blood vessels (H\&E,200X); f: Grade III astrocytoma exhibiting moderate and focal GFAP positivity (GFAP, 200X); g: Grade IV astrocytoma showing increased cellularity, nuclear pleomorphism, vascular proliferation with glomeruloid formation and palisading adjacent to necrosis (H\&E,200X); h: Grade IV astrocytoma exhibiting a few tumor cells with weak GFAP positivity (GFAP, 400X)

In case of ependymomas, GFAP staining was diffuse and more intense in perivascular regions (Fig $2 \mathrm{a} \& \mathrm{~b}$ ) which was consistent with the findings described by Cruz Sanchez et al. ${ }^{21}$ Single case of mixed neuronal glioma and reactive gliosis showed weak focal GFAP positivity in cells exhibiting astrocytic differentiation. In our study, all cases of oligodendroglioma and pituitary adenoma were found to be immunonegative (Fig 2c \& d). Some authors have suggested that oligodendroglial cells do not express GFAP and it may be used as differentiating marker for astrocytoma and oligodendroglioma. ${ }^{2}$ But ultrastructural studies have revealed that gliofibrillary oligodendrocytes and minigemistocytes express high levels of GFAP; perhaps representing transitional form between astrocytic and oligodendrocytic differentiation. ${ }^{22,23}$
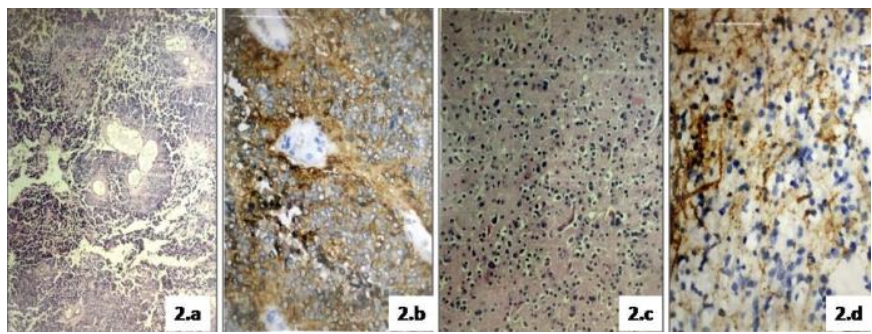

Fig. 2: a: Ependymoma showing rosetting around blood vessel with clear zone (H\&E,100X); b: Ependymoma showing moderate to strong GFAP positivity mainly in clear zone around blood vessels composed of cytoplasmic processes (GFAP, 400X); c: Oligodendroglioma showing increased cellularity, perinuclear halo and chicken wire pattern of proliferating blood vessels (H\&E,200X); d: Oligodendroglioma showing negative GFAP staining in oligodendrocytes and moderate positivity in short processes in astrocytes (GFAP, 200X)

In conclusion, squash technique is an extremely useful tool in the rapid intra-operative diagnosis of gliomas as it is simple, reliable and cost effective. The strength of squash cytology is that it safeguards the surgeon, provides better cellular details with minimum equipment and technical skill; also allows tissue preservation for paraffin embedding for further analysis. However the knowledge of possible errors can minimize misinterpretation and helps in providing a more definitive opinion. Our results corroborate with the opinion that GFAP can be used as an adjunctive marker to verify the 
astroglial differentiation especially in diagnostically difficult cases.

\section{References}

1. Ostrom QT, Bauchet L, Davis FG, Deltour I, Fisher JL, Langer CE et al. The epidemiology of glioma in adults: a "state of the science" review. Neuro Oncol 2014;16(7):896-913.

2. Gupta M, Djalilvand A and Brat DJ. Clarifying the Diffuse Gliomas. An Update on the Morphologic Features and Markers That Discriminate Oligodendroglioma From Astrocytoma. Am J Clin Pathol 2005;124:755-68.

3. Verma SK, Kumar R, Srivani J, Arnold J. Diagnostic Accuracy of Squash Preparations in Central Nervous System Tumors. Iranian Journal of Pathology 2013;8(4):227-34.

4. Cappabianca P, Spaziante R, Caputi F, Pwttinato G, Caro MD, Carrabs G et al. Accuracy of analysis of multiple small fragments of glial tumors obtained by sterotactic biopsy. Acta cytologica 1991;35(5):505-11.

5. Beuvon F, Varlet P, Fallet-Bianco C, DaumasDuport C. The "Smear" technique for the extemporaneous examination; diagnostic contribution to neurosurgical pathology. Ann Pathol 2000;20(5):499-506.

6. Goyal R, Mathur SK, Gupta S, Goyal R, Kumar $\mathrm{S}$, Batra A et al. Immunohistochemical expression of glial fibrillary acidic Protein and CAM5.2 in glial tumors and their role in differentiating glial tumors from metastatic tumors of central nervous system. J Neurosci Rural Pract 2015;6(4):499503.

7. Shrestha S, Thapa BK, Bhattarai B. Smear technique for intraoperative diagnosis of central nervous system neoplasms. Journal of Pathology of Nepal 2014;4:544-47.

8. Rani H, Kulkarni P, Dinesh US, Rao RV, Melkundi S. Comparision of squash and frozen sections versus parrafin sections in the intraoperative diagnosis of central nervous system lesions. Elective Medicine Journal 2013;2(2):1014.

9. Jha BM, Patel V, Patel K, Agarwal A. Role of squash smear technique in intraoperative diagnosis of CNS tumors. Int J Med Sci Public Health 2013;2:889-92.

10. Kumar M, Mitra S, Mukhopadhyay D, Sharma V. Squash preparation: A reliable diagnostic tool in the intraoperative diagnosis of central nervous system tumors. Journal of Cytology 2010;27(3):81-5.

11. Imtiaz AQ, Jamal S, Mamoon N, Luqman M, Sharif MA. Usefulness of crush smears Intraoperative consultation of neurological biopsies: J Coll Physicians Surg Pak 2006;16(9):590-3.

12. Sharma N, Misra V, Singh PA, Gupta SK, Debnath S, Nautiyal A. Comparative Efficacy of Imprint and Squash Cytology in Diagnosing Lesions of the Central Nervous System. Asian Pacific J Cancer Prev 2011;12:1693-6.

13. Kini JR, Jeyraj V, Jayaprakash CS, Indira S, Naik CNR. Intraoperative consultation and smear cytology in the diagnosis of brain tumours.
Kathmandu University Medical Journal 2008;6(24):453-57.

14. Rao S, Rajkumar A, Ehtesham MD, Duvuru P. Challenges in neurosurgical intraoperative consultation. Neurology India 2009;57(4):464-68.

15. Plesec TP, Prayson RA. Frozen Section Discrepancy in the Evaluation of Central Nervous System Tumors. Arch Pathol Lab Med 2007;131:1532-40.

16. Agrawal M, Chandrakar SK, Lokwani D, Purohit MR. Squash Cytology in Neurosurgical Practice: A Useful Method in Resource-Limited Setting with Lack of Frozen Section Facility. Journal of Clinical and Diagnostic Research 2014;8(10):FC09-12.

17. Bignami A, Eng LF, Dahi D, Uyeda CT. Localisation of the glial fibrillary acidic protein in astrocytes by immunoflourescence. Brain Res 1972;43(2):429-35.

18. Velasco ME, Dahl D, Rosessmann U, Gambetti P. Immunohistochemical localisation of glial fibrillary acidic protein in human glial neoplasm. Cancer 1980;45(3):484-94.

19. Chen Y, Zhang YX. Use of monoclonal antibodies to glial fibrillary acidic protein in the cytological diagnosis of brain tumors. Acta cytologica 1989;33(6):922-8.

20. Eng LF, Rubinstein LJ. Contribution of immunohistochemistry to diagnostic problems of human cerebral tumors. J Histochem Cytochem 1978;26(7):513-22.

21. Cruz-Sanchez FF, Ross ML, Hughes JT, Cervos J-Navarro. An immunohistological study of 66 ependymoma. Histopathology 1988;13:443-54.

22. Kros JM, Van Eden CG, Stefanko SZ, Van Batenburg MW, van der Kwast TH. Prognostic implications of glial fibrillary acidic protein containing cell types in oligodendrogliomas. Cancer 1990;66(6):1204-12.

23. Herpers MJ, Budka H. Glial fibrillary acidic protein (GFAP) in oligodendroglial tumors: gliofibrillary oligodendroglioma and transitional oligoastrocytoma as subtypes of oligodendroglioma. Acta Neuropathol 1984;64(4):265-72. 